\title{
Tecnura
}

ESTUDIO DE CASO

\section{Detección de defectos en textiles mediante técnicas espaciales para análisis de textura}

\section{Defect detection in textilles using special techniques fortexture analysis}

\author{
José David Alvarado Moreno
}

Fecha de recepción: 15 de octubre de 2014

Fecha de aceptación: 18 de septiembre de 2015

Cómo citar: Alvarado Moreno, J. D. (2016). Detección de defectos en textiles, utilizando técnicas espaciales para análisis de textura. Revista Tecnura, 20(47), 97-106. doi: 10.14483/udistrital.jour.tecnura.2016.1.a08

\section{Resumen}

En el siguiente trabajo se presenta la utilización de técnicas espaciales de análisis de textura en imágenes para la identificación de defectos en textiles, en donde se presenta la aplicación de técnicas para pre-procesamiento, y para el análisis de textura los patrones locales binarios y la matriz de co-ocurrencia con fin de extraer características resaltar las diferencias e identificar los defectos en textiles haciendo uso del clasificador basado en la distancia de mahalanobis.

Palabras clave: análisis de textura, distancia de mahalanobis, defectos en textiles, matriz de co-ocurrencia, patrones locales binarios, filtrado de imágenes.

\begin{abstract}
This paper presents the use of spatial analysis techniques texture images for defects identification in textiles, where the application of pre-processing techniques is presented, and the analysis of texture local binary patterns and co-occurrence matrix to extract features to highlight differences and identify defects in textiles using classifier based on the Mahalanobis distance.

Keywords: Co-occurrence matrix, defects in textiles, Mahalanobis distance, pre-processing, local binary patterns, texture analysis.
\end{abstract}

Ingeniero Electrónico, magister en Ingeniería de Control Industrial. Docente de la Universidad de Cundinamarca. Fusagasugá, Colombia. Contacto: jdalvarado@mail.unicundi.edu.co 


\section{INTRODUCCIÓN}

El sector textil ha presentado cambios a través de los años, debido a factores como: la demanda, la globalización, la cantidad elevada de productores y cambios en la moda, lo que hace de esta industria un sector receptivo al cambio y en el que la innovación plantea mejoras en los diferentes procesos (Supersociedades, 2013).

En el proceso que las máquinas de hilar fibras realizan, es posible que se puedan presentar diferentes tipos de defectos que generan fallas en el tejido. Por lo general, para su identificación, una persona (operario) hace una inspección visual en busca de dichos defectos. En ocasiones, este tipo de control es insuficiente, pues los imperfectos son demasiado pequeños y no se perciben a simple vista, o porque la fatiga del inspector de calidad impide la identificación (Che-Seung, Byeong-Mook y Moo-Jin, 2005).

Para la inspección automática de los defectos, en el mercado encontramos diferentes soluciones para la industria textil (Ullua y Rodríguez, 2009), en las cuales se han implementado diferentes técnicas de procesamiento de imágenes para la detección de los defectos. En la literatura hay propuestas basadas en la transformada de Fourier (Chi-Ho y Pang, 1999), transformada wavelet (Sungshin, Hyeon, Seong-Pyo y Kwang-Baek,

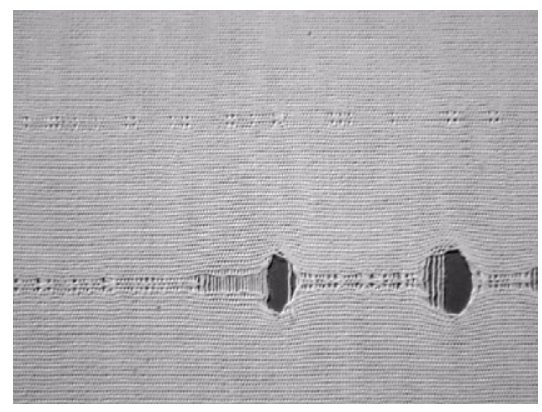

(a) Defecto
2005), demodulación AM-FM (Ullua y Rodríguez, 2009), entre otros.

Anterior a este proyecto se desarrolló un estudio de técnicas de análisis de imágenes para la identificación de defectos en textiles (Fernández y Alvarado, 2010), en donde se clasificaban las imágenes en dos clases: defectos y sin defectos (figura 1); los resultados obtenidos de porcentaje de acierto se muestran en la tabla 1. Las técnicas, matriz de co-ocurrencia (GLCM) y patrones locales binarios (LBP) presentaron mejores resultados en porcentaje de clasificación y en tiempo de procesamiento, comparadas con el análisis de la energía de Laws.

Tabla 1. Resultados obtenidos en la etapa I

\begin{tabular}{ccc}
\hline $\begin{array}{c}\text { Tipo } \\
\text { de Técnica }\end{array}$ & $\begin{array}{c}\text { Porcentaje de } \\
\text { acierto }\end{array}$ & $\begin{array}{c}\text { Tiempo de } \\
\text { procesamiento }\end{array}$ \\
\hline Laws & $82,96 \%$ & $2,064(\mathrm{~s})$ \\
\hline GLCM & $91,32 \%$ & $0,025(\mathrm{~s})$ \\
\hline LBP & $95,52 \%$ & $0,508(\mathrm{~s})$ \\
\hline
\end{tabular}

Fuente: Fernández y Alvarado (2010).

En este artículo se presentan los resultados de la continuación de la evaluación de alternativas de las técnicas espaciales de análisis de textura, en donde se busca identificar los diferentes tipos de defectos que se producen en la fabricación de telas.



(b) Sin Defecto

Figura 1. Imágenes originales base de datos

Fuente: elaboración propia 


\section{METODOLOGÍA}

En este trabajo se utiliza la base de datos texUAN que cuenta con cerca de 2000 imágenes, las cuales representan muestras de tela que no tienen estampados ni adiciones de color y que fueron suministradas por empresas textiles de la ciudad de Ibagué (Tolima). Dicha base de datos está compuesta, originalmente, por imágenes de $640 \times 480$; se elaboró un subconjunto de imágenes de $100 \times 100$ divididas en las siguientes clases de imágenes: pique con 434, barrado con 421, mariposeo con 440, mota tejida con 318, mancha con 342, sin defecto con 1041, que corresponden a los diferentes tipos de defectos de estudio. En la figura 1 se pueden observar las imágenes de $640 \times 480$, y en la figura 2 las diferentes clases de las imágenes de 100×100.

El sistema propuesto se desarrolla en tres etapas: en la primera se analizan diferentes técnicas de filtrado para homogenizar la superficie de las imágenes; en la segunda se estudian técnicas espaciales de análisis de textura, y en la tercera se utiliza un sistema de clasificación y la matriz de confusión para verificar el funcionamiento del sistema de detección de defectos en textiles.

\section{TÉCNICAS DE FILTRADO DE IMÁGENES}

En el proceso de adquisición de las imágenes de la base de datos, el sistema de iluminación utilizado permitió la influencia de luz del entorno con el objetivo de simular un ambiente industrial, debido a esto las imágenes presentan diferencias en contraste y brillo. Para atenuar estas variaciones se buscaron diferentes alternativas para estabilizar los niveles de gris en la imagen.

\section{Filtro basado en la transformada "wavelet"}

Se propone la utilización de un filtro basado en la transformada wavelet, que busca mejorar la distribución de los niveles de gris de la superficie. Se realiza inicialmente un filtro para atenuar los niveles altos de iluminación mediante una wavelet Daubechies; en la caso de los niveles bajos de luminosidad se utiliza la wavelet Haar, donde se descarta la componente de baja frecuencia para obtener un realce de los niveles. En las figuras 3(b) y 3(c) se observa la imagen de salida y la superficie la imagen de la figura 3(a) aplicando esta técnica .

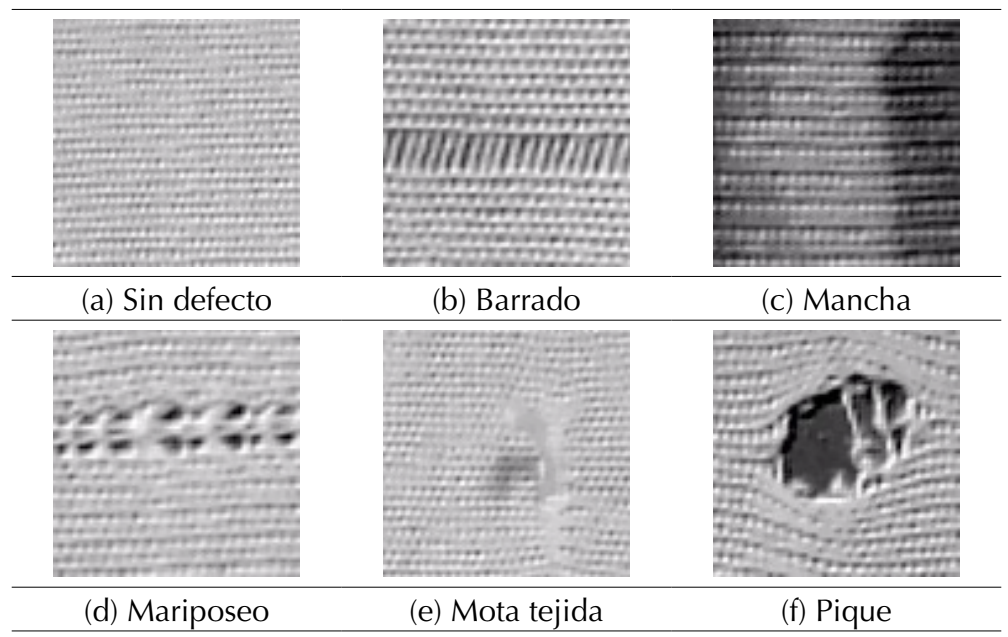

Figura 2. Tipos de imágenes de $100 \times 100$

Fuente: elaboración propia 


\section{Filtro espacial promedio}

Para mejorar tanto la distribución de los niveles de gris de la imagen como la caracterización de la textura, se implementó un filtro espacial utilizando el concepto del filtro promedio. Lo que se plantea inicialmente es el cálculo de la media $(\mu)$ de la imagen, luego se busca que cada pixel esté a menor distancia con respecto a la media. Al aplicar este concepto, se tiene una imagen compacta y corrige algunos problemas de iluminación de la imagen. En la ecuación (1) se define matemáticamente el funcionamiento del filtro y en la figura 3(d) y 3(e) la imagen de salida y la superficie de la figura 3(a) aplicando esta técnica.

\section{TÉCNICAS ESPACIALES DE ANÁLISIS DE TEXTURA}

\section{Matriz de co-ocurrencia (GLCM)}

La técnica matriz de co-ocurrencia analiza las repeticiones de la distribución de los niveles de gris que se presentan en una imagen, en función del pixel de interés y el pixel adyacente denominado vecino; la información recopilada en esta matriz describe la relación de los píxeles en una condición específica de textura.

Para realizar este análisis, la técnica se puede definir en la siguiente secuencia de pasos:

$$
F i(i, j)=\frac{I(i, j)+\mu I}{2} \quad \text { donde } \quad \mu I=\frac{1}{n+m} \sum_{i}^{n} \sum_{j}^{m} I(i, j)
$$

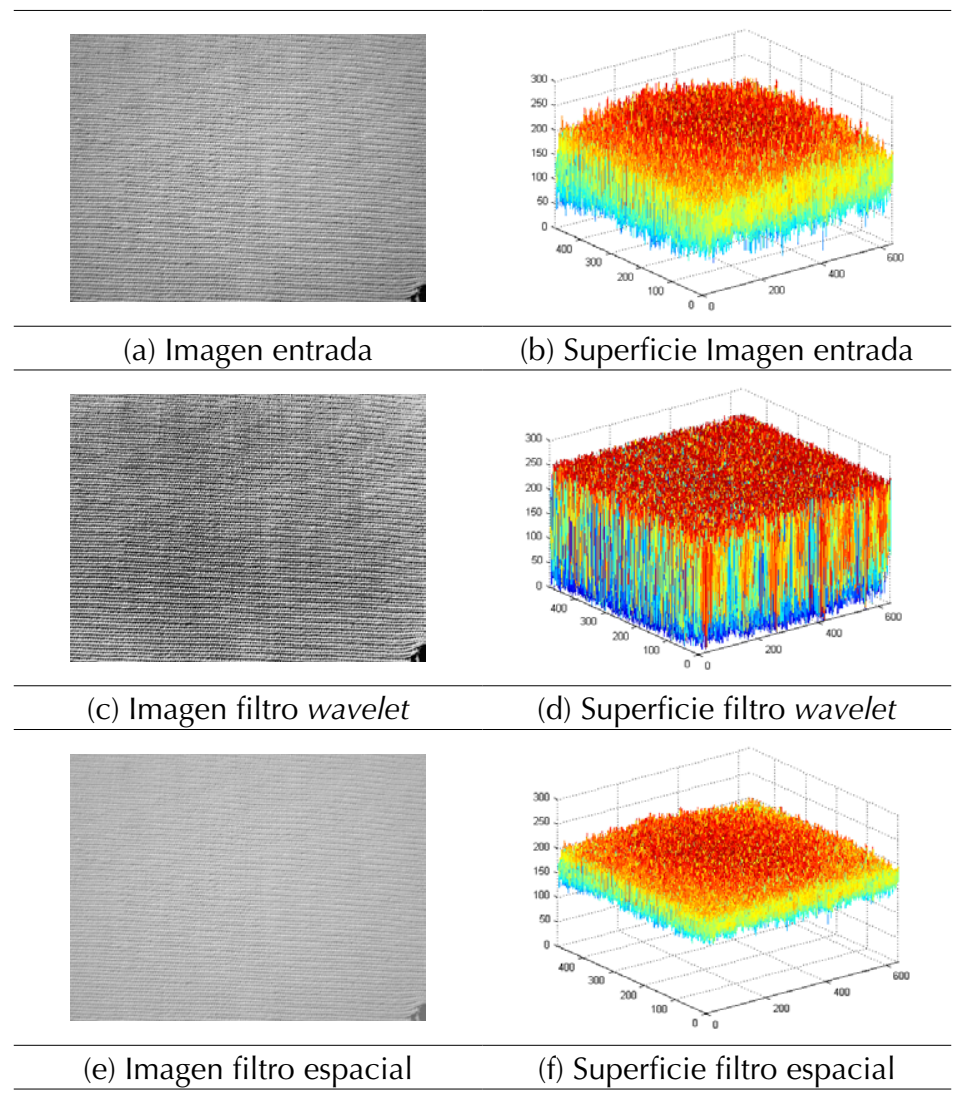

Figura 3. Funcionamiento técnicas de preproceso

Fuente: elaboración propia. 
- La distancia del pixel de interés al vecino.

- El ángulo $(\Theta)$ para establecer el patrón de análisis.

- Los niveles o el número de bits de profundidad de la imagen.

- Recorrer la imagen para recopilar la información en la matriz.

- Utilización de los descriptores.

La distancia espacial interpixel sirve de referencia para el tamaño de la ventana; los valores de esta varían en números impares iniciando en $3 \times 3$ hasta $21 \times 21$ (Haralick, Shanmugam y Dinstein, 1973). El ángulo puede tomar valores de $0^{\circ}$, $45^{\circ}, 90^{\circ}$, y $135^{\circ}$; al variar estos valores es posible caracterizar diferentes tipos de textura o patrones de una textura en particular. En la figura 4 se observa la distribución de los ángulos en una venta de $3 \times 3$.



Figura 4. Distribución de GLCM

Fuente: elaboración propia.

Los niveles de la matriz GLCM se definen como $\mathbf{2}^{\mathbf{n}}$, en donde $\mathbf{n}$ depende de la profundidad de la imagen (número de bits). Por lo general, el análisis de la textura se realiza en imágenes a escala de grises (8 bits), en este caso, el número de niveles es de 256. En la tabla 2 se presenta un ejemplo de la distribución de las posibles combinaciones presentes en una textura de 5 niveles para matriz GLCM. Por lo cual para 256 niveles obtenemos una matriz de tamaño $256 \times 256$ que contiene la información de la distribución espacial, en una distancia y ángulo específico. Por último, se utilizan algunos descriptores de textura (Haralick, 1979) como energía, entropía, contraste, correlación y homogeneidad.

Tabla 2. Matriz de co-ocurrencia

\begin{tabular}{cccccc}
\hline & $\mathbf{0}$ & $\mathbf{1}$ & $\mathbf{2}$ & $\mathbf{3}$ & $\mathbf{4}$ \\
\hline 0 & $(0,0)$ & $(0,1)$ & $(0,2)$ & $(0,3)$ & $(0,4)$ \\
\hline 1 & $(1,0)$ & $(1,1)$ & $(1,2)$ & $(1,3)$ & $(1,4)$ \\
\hline 2 & $(2,0)$ & $(2,1)$ & $(2,2)$ & $(2,3)$ & $(2,4)$ \\
\hline 3 & $(3,0)$ & $(3,1)$ & $(3,2)$ & $(3,3)$ & $(3,4)$ \\
\hline 4 & $(4,0)$ & $(4,1)$ & $(4,2)$ & $(4,3)$ & $(4,4)$ \\
\hline
\end{tabular}

Fuente: elaboración propia.

\section{Patrones locales binarios (LBP)}

El operador de análisis de textura LBP está definido como una medida invariante a la escala de grises, derivado de una definición general de la textura en una vecindad local (Alvarado y Fernández, 2010). La propuesta original (Ojala, Pietikäinen y Harwood, 1999) consiste en la comparación de pixel central con los vecinos, en donde el pixel central es tomado como el umbral con respecto a sus vecinos. Al comparar el pixel central con el vecino se le asigna un valor de uno (1) si el vecino es mayor o igual; en caso contrario, se le asigna un valor de cero (0). A cada resultado del umbral se le asigna un peso de $2^{n}$, en donde $n$ depende de la posición del vecino con respecto al pixel central. Por último, se realiza una suma de los diferentes pesos obteniendo la representación LBP de pixel.

El funcionamiento del operador original se presenta en la figura 5 , donde se analiza una ventana de tamaño $3 \times 3$ y la relación del pixel central en esta caso con sus 8 vecinos. El equivalente LBP para los valores de la figura $5(b)$ se presentan en la ecuación (2).

$$
L B P=(0 * 1)+(1 * 2)+(1 * 4)+(1 * 8)+(2)(1 * 16)+(0 * 32)+(0 * 64)+(1 * 128)=158
$$




\begin{tabular}{|l|l|l|}
\hline 50 & 95 & 85 \\
\hline 72 & 50 & 92 \\
\hline 45 & 87 & 80 \\
\hline
\end{tabular}

(a) Muestra

\begin{tabular}{|l|l|l|}
\hline 0 & 1 & 1 \\
\hline 0 & & 1 \\
\hline 0 & 1 & 1 \\
\hline
\end{tabular}

(b) Patrón



(c) Pesos
Figura 5. Funcionamiento del operador LBP

Fuente: elaboración propia.

Posteriormente, del operador LBP original se deriva uno nuevo, que modifica los tamaños de la ventana y el número de vecindades para pixel de interés (Mäenpää, 2003). Para esta derivación se definen unos parámetros denominados $R$ y $P$, donde $R$ corresponde a la distancia (radio) a partir del pixel de origen al vecino y $P$ el número de vecinos utilizados para el cálculo de LBP.

La distancia y la distribución de los vecinos $P$ se encuentran igualmente espaciados y repartidos en una circunferencia simétrica que se construye a partir del valor de $R$, para valores de $R>0$ y $P>1$. Al modificar $P$ y $R$ se pueden obtener diferentes medidas de textura del área de interés. En la figura 6 se puede observar la distribución de los vecinos para diferentes valores de $R$ y $P$.

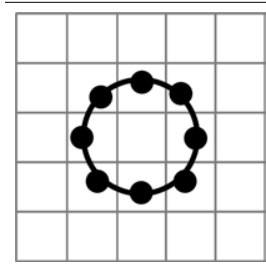

(a) $R=1, P=8$

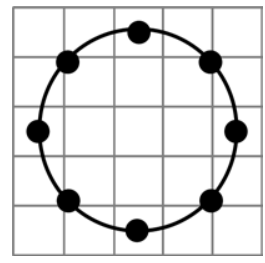

(b) $R=2, P=8$

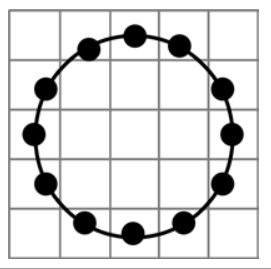

(c) $R=2, P=12$
Figura 6. Funcionamiento del operador LBP

Fuente: elaboración propia.

La distribución circular de los pixeles vecinos se realiza mediante la ecuación (3), con la que se establece una aproximación inicial de la ubicación de los vecinos, a lo que es necesario complementarlo con un proceso de interpolación para definir las coordenadas (x.y) de los vecinos:

$$
x=R \operatorname{Sen}\left(\frac{2 \pi p}{P}\right), y=R \operatorname{Cos}\left(\frac{2 \pi p}{P}\right),
$$

Por último, la derivación del operador LBP para una imagen se define en la ecuación (4).

$$
L B P_{P, R}\left(x_{c}, y_{c}\right)=\sum_{P=0}^{P-1} s\left(g_{c}-g_{c}\right) 2^{P}
$$

\section{Patrones locales binarios invariantes a la rotación}

El trabajo de Ojala, Pietikainen y Mäenpää (2002) plantea un operador derivado del modelo $L B P_{P, R^{\prime}}$ en este se considera cada pixel como un centro de giro y se incorpora el concepto de la rotación invariante local utilizando la forma circular de la relación con sus vecinos; se establecen patrones de textura definidos de la forma circular del operador, que analiza las diferencias de textura debido a cambios en las posiciones relativas de una fuente de luz y el objeto de análisis. No considera los cambios producidos por los elementos de captura que son causados por los efectos de digitalización. Para este operador, el parámetro $P$, además del número de vecinos, define los niveles del operador, en el caso de $P=8$, el valor cada LBP tiene 8-bits, lo que representa 256 niveles y 36 únicos patrones locales de invariantes en su rotación (Ojala, Pietikainen y Mäenpää, 2002). En la figura 7 se ilustran seis tipos de patrones de texturas invariantes en su rotación.

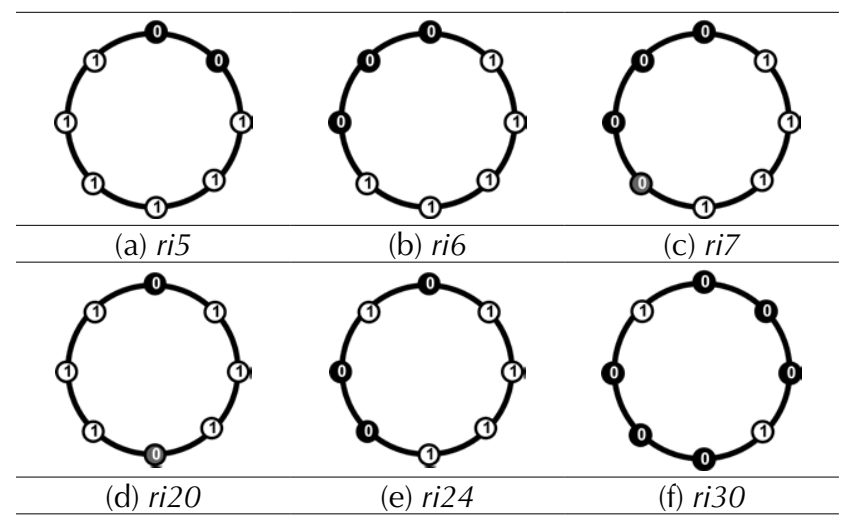

Figura 7. Patrones locales invariantes en la rotación para $P=8$

Fuente: elaboración propia. 
El operador $B P_{8, R}^{r i 36}$ para $P=8$ se puede representar como un histograma de 36 distribuciones, en donde se resumen las repeticiones de los diferentes patrones ri36, matemáticamente se define en la ecuación (5).

$$
\operatorname{LBP} P_{8, R}^{r i 36}=\min \left\{R O R\left(L B P_{8, R}\right)\right\}
$$

Donde $\operatorname{ROR}\left(L B P_{8, R}\right)$ realiza una rotación bit a bit de cada patrón ri36; el número de rotaciones posibles puede variar dependiendo del cada patrón entre 0 a 7 . En la figura 8 se observa el funcionamiento para ri7.

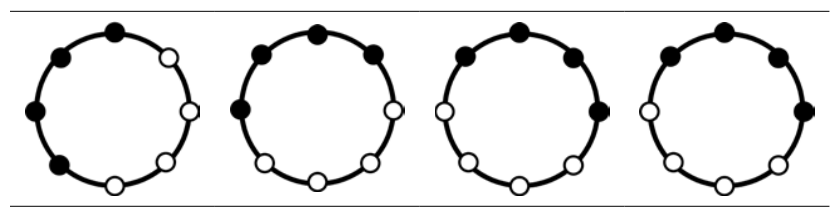

(a) $\operatorname{rit}(1)$

(b) $\operatorname{ri7}(2)$

(c) $r i 7(3)$

(d) $r i 7(4)$

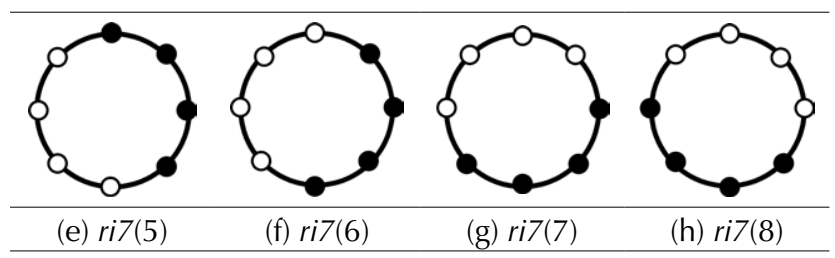

Figura 8. Funcionamiento de $R O R\left(L B P_{8, R}\right)$ para ri7

Fuente: elaboración propia.

El operador $L B P^{r i 36}$ posteriormente se le incorpora un concepto adicional de la uniformidad, en donde se realiza una división de los tipos de patrones definidos originalmente en el operador; este establece dos clases (Topi, Timo, Matti y Maricor, 2000): la primera compuesta de 9 texturas que forman un subconjunto de las texturas denotadas como uniformes; el segundo subconjunto conformado por las 27 texturas restantes denominadas no uniformes. Esta metodología se considera una descripción robusta y eficiente que puede alcanzar mejores índices de clasificación. Para este operador se obtiene un histograma de 10 distribuciones las cuales corresponden 9 al primer subconjunto y las restantes 27 a la distribución faltante; el operador LBP invariante en la rotación uniforme se denota en la ecuación (6):

$$
L B P_{8, R}^{r i u 2}=\left\{\begin{array}{cc}
\sum_{P=0}^{P-1} s\left(g_{c}-g_{c}\right) 2^{P} & \text { ri36 } \leq 9 \\
9 & \text { ri36 }>9
\end{array}\right.
$$

\section{MÉTODO DE CLASIFICACIÓN}

Para el sistema de clasificación se utiliza el concepto de la distancia de Mahalanobis (1936), que es usado frecuentemente para medir la distancia entre un individuo y el centro de la población de donde procede el individuo. Este concepto permite establecer la semejanza de un individuo con el resto de la población (Mahalanobis, 1936), cada patrón seleccionado aleatoriamente se define como un vector $x_{i}=\left(x_{i(1)} x_{i(2)}, \ldots, x_{i(n)}\right)$, para una población con centro $\mu_{i}=\left(\mu_{i(1)} \mu_{i(2)}, \ldots ., \mu_{i(n)}\right)$, y una matriz de covarianzas $\sum$.

El sistema propuesto busca establecer si la imagen presenta algún defecto y a qué tipo de defecto corresponde. Las clases establecidas son: (1) sin defecto, (2) barrado, (3) mancha, (4) mariposeo, (5) mota tejida y (6) pique. Al presentar una imagen de entrada aleatoria, el proceso de clasificación consiste en una fase de aprendizaje y una fase de clasificación.

\section{Fase de aprendizaje}

Se toman $N$ imágenes que pertenecen a cada clase; a cada imagen se le extrae el vector característico $x_{i}$ definido por el tipo de técnica de análisis de textura; cada vector se recopila en una matriz $M$ representada en la ecuación (7).

Se le calcula a $M$ el vector de media $\mu_{i}$ y la matriz inversa de covarianza $\Sigma^{-1}$ para cada clase.

Se almacenan los vectores de media $\mu_{i}$ y la matriz inversa de covarianza $\Sigma^{-1}$ para cada clase. 


$$
M(i, j)=\left[\begin{array}{cccc}
x_{i}(1,1) & x_{i}(1,2) & \ldots & x_{i}(1, j) \\
x_{i}(2,1) & x_{i}(2,2) & \ldots & x_{i}(2, j) \\
\vdots & \vdots & & \ddots \\
x_{i}(i, 1) & x_{i}(i, 2) & \ldots & x_{i}(i, j)
\end{array}\right]
$$

\section{Fase de clasificación}

En este paso se realiza también la extracción del vector de características de cada imagen a clasificar $x_{i}$.

Se calcula la distancia de Mahalanobis $d_{i}$ entre el vector de características $x_{i^{\prime}}$ el vector media $\mu_{i}$ y la matriz inversa de covarianza $\Sigma^{-1}$, en donde obtenemos un vector $D=\left[d_{i}(1), d_{i}(2), d_{i}(3), d_{i}(4)\right.$,$\left.d_{i}(5), d_{i}(6)\right]$ que corresponde a las distancias de la imagen de entrada con cada clase.

$$
d_{i}=\left(x_{i}-\mu_{i}\right)^{T} \Sigma^{-1}\left(x_{i}-\mu_{i}\right)
$$

Por último, se incorporó un algoritmo de competencia para determinar la pertenencia de los datos a una clase determinada. De esta manera es posible establecer la clase a la que pertenece una imagen para los diferentes tipos de defectos establecidos.

\section{RESULTADOS}

Para la validación de los resultados se utilizaron las imágenes de la base de datos texUAN, donde $50 \%$ de las imágenes de cada clase son usadas para el entrenamiento, y el porcentaje restante, para la clasificación. Para establecer los porcentajes de acierto se utilizó el concepto de matriz de confusión (Umbaugh, 2010) para las seis clases establecidas. En la tabla 3 se muestra la matriz de confusión utilizando el filtro promedio y la técnica $L B P_{8, R}^{r i 36}$

Los resultados obtenidos para la técnica de patrones locales binarios (LBP) y sus extensiones se resumen en la tabla 4 ; las imágenes sin ningún tipo de filtrado, en la tabla 5, y en la tabla 6 se muestran los resultados aplicando los dos tipos de filtrado descritos.
Tabla 3. Matriz de confusión para $L B P_{8, R}^{r i 36}$ con filtro espacial

\begin{tabular}{ccccccc}
\hline & C1 & C2 & C3 & C4 & C5 & C6 \\
\hline C1 & $100 \%$ & $0 \%$ & $0 \%$ & $0 \%$ & $0 \%$ & $0 \%$ \\
\hline C2 & $0 \%$ & $88,4 \%$ & $4,8 \%$ & $2,0 \%$ & $2,8 \%$ & $2,0 \%$ \\
\hline C3 & $0 \%$ & $0 \%$ & $84,2 \%$ & $6,3 \%$ & $8,9 \%$ & $0,5 \%$ \\
\hline C4 & $0 \%$ & $1,4 \%$ & $1,4 \%$ & $91,4 \%$ & $4,3 \%$ & $1,4 \%$ \\
\hline C5 & $3,3 \%$ & $0 \%$ & $1,1 \%$ & $0 \%$ & $94,4 \%$ & $1,1 \%$ \\
\hline C6 & $0,9 \%$ & $0,4 \%$ & $2,2 \%$ & $0,9 \%$ & $5,2 \%$ & $90,4 \%$ \\
\hline
\end{tabular}

Fuente: elaboración propia.

Tabla 4. Resultados LBP sin filtrado.

\begin{tabular}{cccc}
\hline & $L B P 8,1$ & $L B P_{8, R}^{\text {ri36 }}$ & $L B P_{8, R}^{\text {riu2 }}$ \\
\hline C1 & $42,0 \%$ & $99,0 \%$ & $82,0 \%$ \\
\hline C2 & $13,2 \%$ & $89,2 \%$ & $66,8 \%$ \\
\hline C3 & $37,4 \%$ & $86,8 \%$ & $67,4 \%$ \\
\hline C4 & $30,0 \%$ & $81,4 \%$ & $31,4 \%$ \\
\hline C5 & $46,7 \%$ & $90,0 \%$ & $77,8 \%$ \\
\hline C6 & $57,0 \%$ & $94,3 \%$ & $47,4 \%$ \\
\hline
\end{tabular}

Fuente: elaboración propia

Tabla 5. Resultados LBP filtro wavelet

\begin{tabular}{cccc}
\hline & $L B P 8,1$ & $L B P_{8, R}^{r i 36}$ & $L B P_{8, R}^{r i u 2}$ \\
\hline C1 & $56,0 \%$ & $93,0 \%$ & $57,0 \%$ \\
\hline C2 & $13,2 \%$ & $84,2 \%$ & $55,2 \%$ \\
\hline C3 & $41,6 \%$ & $81,6 \%$ & $42,1 \%$ \\
\hline C4 & $27,1 \%$ & $97,1 \%$ & $51,4 \%$ \\
\hline C5 & $46,7 \%$ & $96,7 \%$ & $74,4 \%$ \\
\hline C6 & $46,1 \%$ & $87,4 \%$ & $41,4 \%$ \\
\hline
\end{tabular}

Fuente: elaboración propia.

Tabla 6. Resultados LBP filtro espacial

\begin{tabular}{cccc}
\hline & $L B P 8,1$ & $L B P_{8, R}^{r i 36}$ & $L B P_{8, R}^{\text {riu2 }}$ \\
\hline C1 & $55,0 \%$ & $100 \%$ & $81,0 \%$ \\
\hline C2 & $17,2 \%$ & $88,4 \%$ & $61,6 \%$ \\
\hline C3 & $46,8 \%$ & $84,2 \%$ & $68,4 \%$ \\
\hline C4 & $34,3 \%$ & $91,4 \%$ & $40,0 \%$ \\
\hline C5 & $46,7 \%$ & $94,4 \%$ & $71,1 \%$ \\
\hline C6 & $42,2 \%$ & $90,4 \%$ & $56,1 \%$ \\
\hline
\end{tabular}

Fuente: elaboración propia. 
En el caso de la matriz de co-ocurrencia (GLCM), se analizaron los resultados para las posibles combinaciones de GLCM $0^{\circ}$, GLCM 45, GLCM $90^{\circ}$ y GLCM 135, y cada uno de estos, para un total 15 posibilidades. En la tabla 7 se muestran las cuatro opciones que presentan mayores porcentajes de acierto, mediante la técnica de filtrado basada en el filtro wavelet que presentó mejores resultados para esta técnica.

Tabla 7. Resultados GLCM filtro wavelet

\begin{tabular}{|c|c|c|c|c|}
\hline & $\begin{array}{c}\text { GLCM } \\
9^{\circ} \\
\end{array}$ & $\begin{array}{l}\text { GLCM } \\
0^{\circ}, 9^{\circ} \\
\end{array}$ & $\begin{array}{c}\text { GLCM }^{\circ} \\
45^{\circ}, 90^{\circ}\end{array}$ & $\begin{array}{c}\text { GLCM } 0^{\circ} \\
45^{\circ}, 90^{\circ}, 135^{\circ}\end{array}$ \\
\hline C1 & $63,0 \%$ & $67,0 \%$ & $79,0 \%$ & $79,0 \%$ \\
\hline $\mathrm{C} 2$ & $38,0 \%$ & $47,6 \%$ & $58,0 \%$ & $70,8 \%$ \\
\hline $\mathrm{C} 3$ & $50,0 \%$ & $63,2 \%$ & $61,6 \%$ & $66,8 \%$ \\
\hline $\mathrm{C} 4$ & $52,9 \%$ & $58,6 \%$ & $77,1 \%$ & $80,0 \%$ \\
\hline C5 & $41,1 \%$ & $84,4 \%$ & $87,8 \%$ & $89,9 \%$ \\
\hline C6 & $63,9 \%$ & $74,3 \%$ & $83,5 \%$ & $87,8 \%$ \\
\hline
\end{tabular}

Fuente: elaboración propia.

\section{CONCLUSIONES}

Las técnicas de filtrado utilizadas mejoran el porcentaje de acierto de las técnicas de análisis de textura. Para los patrones locales binarios el filtro promedio y en la matriz de co-ocurrencia el filtro wavelet, incrementaron el porcentaje de acierto en la identificación de los defectos en la tela. En la identificación de los diferentes defectos de estudio el filtro wavelet aumenta el porcentaje de acierto en la clasificación del mariposeo y la mota tejida, el filtro promedio en imágenes sin defecto, y sin utilizar filtros el barrado, la mancha y el pique.

Para la matriz de coocurrencia se exploraron deferentes alternativas para mejorar el porcentaje de acierto, dentro de las cuales la que mostró mejores resultados de clasificación fue la combinación de las cuatro componentes de $\operatorname{GLCM~} 0^{\circ}, 45^{\circ}$, $90^{\circ}, 135^{\circ}$. En resumen, la técnica no resultó ser tan eficiente para detección de los diferentes defectos textiles de las imágenes analizadas.
La derivación original del operador LBP no fue eficiente para la identificación de defectos en textiles debido a que la información recopilada es una descripción general de la textura, y las características de las imágenes de estudio presentan una similitud que no se puede establecer con este método. Por otra parte las extensiones derivadas de $L B P^{r i 36}$ y $L B P^{r i u 2}$ presentaron mejores porcentajes en la clasificación, donde ri36 resultó ser la técnica con mejores resultados en la identificación de defectos en textiles, ya que a través de esta se busca caracterizar las texturas uniformes y no uniformes presentes en la imagen.

\section{FINANCIAMIENTO}

Este proyecto fue financiado por la Universidad Antonio Nariño en la convocatoria interna para proyectos de investigación.

\section{REFERENCIAS}

Alvarado, J.D. \& Fernández, J. (2012). Análisis de textura en imágenes a escala de grises, utilizando patrones locales binarios (LBP). Revista ENGI 1(1), 1-6. Recuperado de: http://revistas_electronicas. unicundi.edu.co/index.php/Revistas_electronicas/ article/view/5/5

Che-Seung, C.; Byeong-Mook, C. \& Moo-Jin, P. (2005, Aug). Development of Real-Time Vision-Based Fabric Inspection System. Industrial Electronics, IEEE Transactions on 52(4), 1073-1079. DOI:10.1109/ TIE.2005.851648

Chi-Ho, C. \& Pang, G. (1999). Fabric defect Detection by Fourier Analysis. Industry Applications Conference, 3, 1743-1750. DOI: 10.1109/IAS.1999.805975

Fernandez, J. \& Alvarado, J.D. (2010). Estudio comparativo de técnicas espaciles para la identificacion de defectos en textiles. ITECKNE 7(1), 75-82. Recuperado de: http://revistas.ustabuca.edu.co/index.php/ ITECKNE/article/download/354/316

Haralick, R.M. (1979). Statistical and Structural Approaches to Texture. Proceedings of the IEEE 67(5), 786804. DOI:10.1109/PROC.1979.11328 
Haralick, R.; Shanmugam, K. \& Dinstein, I. (1973). Texture Features for Image Classification. Systems, Man and Cybernetics, SMC, 3, 610-621. DOI:10.1109/ TSMC.1973.4309314

Mäenpää, T. (2003). The Local Binary Pattern Approach to Texture Analysis-Extensions and Applications. Dissertation, Acta Univ Oul C 187, 78 p + App.: University of Oulu.

Mahalanobis, P. C. (1936). On the Generalized Distance in Statistics. Proceedings of the National Institute of Sciences II(1), 49-55.

Ojala, T.; Pietikäinen, M. \& Harwood, D. (1999). A Comparative Study of Texture Measures with Classification Based on Featured Distributions. Pattern Recognition 29(1), 51-59. DOI:10.1016/0031-3203(95)00067-4.

Ojala, T.; Pietikainen, M. \& Maenpaa, T. (2002). Multiresolution Gray-Scale and Rotation Invariant Texture Classification with Local Binary Patterns. Pattern Analysis and Machine Intelligence, IEEE Transactions on 24(7), 971-987. DOI:10.1109/ TPAMI.2002.1017623
Sungshin, K.; Hyeon, B.; Seong-Pyo, C. \& KwangBaek, K. (2005). On-line Fabric-Defects Detection Based on Wavelet Analysis. En ICCSA. Computational Science and Its Applications, 3483, 1075-1084). Berlín: Springer Berlin Heidelberg. DOI:10.1007/11424925

Superintendencia de Sociedades. (2013). Desempeño del sector textil confeccion 2008-2012. Bogotá D.C.: Delegatura de Asuntos Económicos y Contables, Grupo de Estudios Económicos y Financieros. Topi, M.; Timo, O.; Matti, P. \& Maricor, S. (2000). Robust Texture Classification by Subsets of Local Binary Patterns. Pattern Recognition, 2000. Proceedings. 15th International Conference on 3, 935938. doi:10.1109/ICPR.2000.903698

Ullua, A. \& Rodríguez, P. (2009). Detección automática de defectos en telas basado en la demodulación AM-FM. Ibero-American Conference on Trends in Engineering Education and Collaboration.

Umbaugh, S. E. (2010). Digital Image Processing and Analysis: Human and Computer Vision Applications with CVIPtools. Boca Raton: CRC Press. 